Running head: RESPONSE INHIBITION IN MEN WITH PTSD/ADHD

\title{
Cue-Dependent Inhibition in Posttraumatic Stress Disorder and Attention-
}

\section{Deficit/Hyperactivity Disorder}

\author{
Zachary W. Adams, Ph.D. ${ }^{\mathrm{a}, \mathrm{b}}$ \\ Michael Meinzer, Ph.D. ${ }^{\mathrm{c}}$ \\ Howard Mandel, B.S. ${ }^{d}$ \\ Joshua Voltin, B.S. ${ }^{\mathrm{b}}$ \\ Blaine Caughron, M.S. ${ }^{\mathrm{d}}$ \\ Floyd R. Sallee, MD ${ }^{\mathrm{d}}$ \\ Mark Hamner, MD ${ }^{\text {b, d }}$ \\ Zhewu Wang, MD*b, d
}

*Please send correspondence to: Zhewu Wang, MD, Ralph H. Johnson VA Medical

Center/Medical University of South Carolina, 109 Bee Street, Charleston, SC. USA. Phone:

843-789-7949; Fax: 843-792-3195. Email: wanzh@musc.edu

\footnotetext{
a Indiana University School of Medicine, Indianapolis, IN, USA

${ }^{\mathrm{b}}$ Medical University of South Carolina, Charleston, SC, USA

${ }^{\mathrm{c}}$ University of Maryland, College Park, MD, USA

${ }^{\mathrm{d}}$ Ralph H. Johnson VA Medical Center, Charleston, SC, USA
}

This is the author's manuscript of the article published in final edited form as:

Adams, Z. W., Meinzer, M., Mandel, H., Voltin, J., Caughron, B., Sallee, F. R., ... Wang, Z. (2017). Cue-Dependent Inhibition in Posttraumatic Stress Disorder and Attention-Deficit/Hyperactivity Disorder. Journal of Anxiety Disorders, 51, 1-6. https://doi.org/10.1016/j.janxdis.2017.08.003 
Highlights

- Response inhibition was compared in 161 adult men with PTSD and/or ADHD and controls.

- PTSD and ADHD+PTSD were linked to impaired response execution and inhibition.

- PTSD and ADHD+PTSD showed slower, more variable reaction time relative to controls.

- No cue dependency differences were observed by PTSD or ADHD diagnosis.

\begin{abstract}
Objective: Attention-deficit/hyperactivity disorder (ADHD) and posttraumatic stress disorder (PTSD) are common among military veterans, but the comorbidity of these two psychiatric disorders remains largely unstudied. Evaluating response inhibition and cue-dependent learning as behavioral and neurocognitive mechanisms underlying ADHD/PTSD can inform etiological models and development of tailored interventions.
\end{abstract}

Method: A cued go/no-go task evaluated response inhibition in 160 adult males. Participants were recruited from the community and a Veterans Administration medical center. Four diagnostic groups were identified: ADHD-only, PTSD-only, ADHD+PTSD, controls.

Results: Group differences were observed across most indices of inhibitory functioning, reaction time, and reaction time variability, whereby PTSD-only and ADHD+PTSD participants demonstrated deficits relative to controls. No cue dependency effects were observed.

Conclusion: Findings complement prior work on neurocognitive mechanisms underlying ADHD, PTSD, and ADHD+PTSD. Lack of expected group differences for the ADHD-only group may be due to limited power. Additional work is needed to better characterize distinctions among clinical groups, as well as to test effects among women and youth.

Keywords: ADHD; PTSD; comorbidity; response inhibition; cued reaction time task; executive functioning

\title{
Abstract
}


Conclusion: Findings complement prior work on neurocognitive mechanisms underlying ADHD, PTSD, and ADHD+PTSD. Lack of expected group differences for the ADHD-only group may be due to limited power. Additional work is needed to better characterize distinctions among clinical groups, as well as to test effects among women and youth. 


\section{Cue-Dependent Inhibition in Posttraumatic Stress Disorder and Attention- Deficit/Hyperactivity Disorder}

Post-traumatic stress disorder (PTSD) is a commonly occurring and debilitating condition following exposure to life-threatening events (e.g., physical assault, sexual assault, combat exposure). Epidemiological studies suggest that even though the vast majority of US citizens experience a traumatic event in their lifetime (Breslau, 2009), the 12-month prevalence of PTSD in US adults is only $3.5 \%$. However, this rate is substantially higher among veterans. For example, $48.5 \%$ of Operation Enduring Freedom (OEF) or Operation Iraqi Freedom (OIF) soldiers screened positive for PTSD symptoms (Khaylis, 2011). Further, $46 \%$ of active duty or retired OEF/OIF soldiers met diagnostic criteria for PTSD (Pittman, 2012). In addition to the high rates of PTSD in veterans, lifetime comorbidity of PTSD with any psychiatric disorder (e.g., depression, substance use disorders, anxiety disorders) has been found to be as high as $88 \%$.

Attention-deficit/hyperactivity disorder (ADHD) has also been shown to commonly occur among military personnel with estimates at approximately 10\% (Antshel, 2013; Hanson, 2012), whereas estimates of adult ADHD in the general population range from 1.6-4.4\% (Kessler, 2006; Simon, 2009). ADHD is estimated to co-occur with PTSD at rates ranging from of 12-28\% in veterans (Adler, 2004; Harrington, 2012). Rates of PTSD among adults with ADHD were six times higher than among adults without ADHD (Antshel, 2013). Furthermore, research has demonstrated that ADHD symptom severity significantly predicts PTSD symptom severity (Harrington, 2012).

Though research has begun to uncover the increased risk for PTSD among individuals with ADHD, less is known about the mechanisms by which these two disorders co-occur. Neurocognitive performance represents one potential area that may help to explain ADHD- 
PTSD comorbidity. Given that both ADHD and PTSD literatures individually identify deficits in neurocognitive performance (Barkley, 1997; Bremner, 1999; Buckley, 2000), investigating specific facets of neurocognitive functioning signifies a promising line of research. Understanding neurocognitive performance among people with PTSD, ADHD, comorbid ADHD-PTSD, and healthy controls will help in building more sophisticated etiological models of the disorders, and help guide targeted treatments that address underlying deficits.

One aspect of neurocognition that has been routinely explored in individuals with ADHD is response inhibition or the inhibition of prepotent or ongoing responses (Barkley, 1997; Oosterlan, 1998). This deficit in response inhibition and interference control has been suggested as a basis for the core features of ADHD (Barkley, 1997). Response inhibition has been routinely measured using the "Go/No Go" Task in which there are two stimuli, a "go" and a "nogo." Participants are then instructed to press a button, as quickly as possible, when a "go" stimulus is presented and to inhibit this response when a "no-go" stimulus is presented (Simmonds, 2008). Research suggests adults with ADHD show lower accuracy rates, increased commission errors, increased response interference, and a decreased likelihood to consciously detect these errors (Morein-Zamir, 2014; O’Connell, 2009; Sebastian, 2012; Woltering, 2013).

Response inhibition also has been investigated, albeit to a lesser degree, within the PTSD literature. According to the DSM-5, PTSD is comprised of four clusters of symptoms: reexperiencing symptoms, avoidance, negative cognitions/mood, and arousal (American Psychiatric Association, 2013). Kertzman and colleagues (2014) pointed to re-experiencing symptoms as well as sensory stimuli leading to cognitive deficits such as response inhibition in patients with PTSD. Self-reported behavioral inhibition is significantly related to PTSD symptoms, especially PTSD symptoms in the avoidance cluster among veterans (Myers, 2012). 
Research using the Go/No-Go task has demonstrated that veterans with PTSD show a decreased inhibitory response and greater false-alarm rate (Tillman, 2010) as well as a significantly more variable reaction times (Swick, 2013) when compared to veterans without PTSD. Several studies have investigated behavioral and neural processing deficits in both inhibitory functioning and evaluation of contextual cues in veterans and civilians with PTSD, in line with evidence that problems in inhibiting a fear response and interpreting environmental safety vs. danger information may underlie symptoms of the disorder (Falconer, 2012; Jovanovic, 2012; Jovanovic, 2013; van Rooij, 2015). Results from those studies suggest that behavioral and neural deficits in inhibition and contextual cue processing may extend beyond fear responses in PTSD and may be more generalized, core deficits of the disorder (Jovanovic 2012; Jovanovic 2013; van Rooij, 2014; van Rooij, 2015). Although some of the studies evaluated the potential effects of comorbid psychiatric conditions (e.g., mood disorders), none directly reported on the potential influence of ADHD on observed group differences.

Little is known about the inhibitory functioning among individuals with ADHD and comorbid PTSD. To our knowledge there have been no studies that have looked at response inhibition in groups of veterans and civilians with ADHD, PTSD, and comorbid ADHD-PTSD compared to veterans and civilians without a history of psychopathology. It is unknown whether people with comorbid ADHD-PTSD have worse inhibitory functioning than people with either disorder alone.

When evaluating inhibitory responses, it is important to consider the potential influence of environmental factors, such as cues that may guide response preparation. Given the symptoms seen in PTSD, especially those related to arousal and hypervigilance (American Psychiatric Association, 2013), veterans with PTSD may be especially attuned to environmental cues to 
inform behavioral control (e.g., vigilance for danger cues). Some evidence suggests that valid cues can aid in the anticipation of an appropriate response against inhibitory impairment. For example, in alcohol use literature, antecedent cues during the Go/No-Go task can help dampen inhibitory deficits when individuals are under the influence of alcohol (Marczinski, 2005). However, the use of antecedent cues during the Go/No-Go task has not been shown to significantly improve response inhibition deficits in adults with ADHD (Roberts, 2016). It is unknown whether having a diagnosis of PTSD would influence cue dependency among veterans and community adults with and without comorbid ADHD.

The current study seeks to investigate how response inhibition is influenced by diagnoses of ADHD, PTSD, or comorbid ADHD-PTSD in veterans and civilians compared to healthy controls. Furthermore, the study will examine how antecedent cues effect response inhibition in these groups. We predicted that participants with ADHD - with and without PTSD - would demonstrate inhibitory deficits relative to controls. We also hypothesized that participants with PTSD would demonstrate greater cue dependency than participants without PTSD.

\section{Method}

\section{Participants}

Participants in this study were 160 men (mean age $=30.8$ years, $\mathrm{SD}=7.6 ; 75.2 \%$ white/Caucasian; $14.9 \%$ black/African-American; 3.0\% biracial; 3.1\% Hispanic). Participants were either recruited from the local community or through a large Veterans Administration (VA) Medical Center in the southeastern U.S. Participants enrolled from the VA were combat veterans of Operation Iraqi Freedom/Operation Enduring Freedom (OIF/OEF) missions. Of enrolled participants, 17 met criteria for ADHD only (47\% veterans), 25 met criteria for PTSD only (100\% veterans), 26 met criteria for ADHD+PTSD (100\% veterans), and 93 participants 
met criteria for neither ADHD nor PTSD (23\% veterans). Basic demographic characteristics for each group are summarized in Table 1.

Inclusion and exclusion criteria. To be eligible for this study, participating veterans were required to have a history of combat exposure, as evidenced by formal release or discharge paperwork (i.e., DD Form 214), a report of combat exposure during the interview with a psychiatrist [masked], and a minimum score of 10 on the Combat Exposure Scale (CES) (Lund, 1984). Participants were not required to meet diagnostic criteria for PTSD or ADHD to enroll, and participants with major depression, and anxiety disorders were included. Subjects with other Axis I psychiatric disorders were excluded from this study; this included current or lifetime DSM-IV schizophrenia, other psychotic disorders, bipolar disorder, and active substance abuse or dependence in the past six months. Individuals with a past history of substance abuse and dependence were included if the last use of the substance was over 6 months prior to the enrollment. There were no inclusion or exclusion criteria based on sociodemographic characteristics.

\section{Procedure}

The study protocol was approved by the Institutional Review Board (IRB) of the academic institution where this research was conducted. A brief description of the study, including explanation of the voluntary nature of participation, was given to potential participants by a trained research assistant. People who expressed interest in participation were screened to determine eligibility for study involvement using the inclusion and exclusion criteria described below. Institutionally approved informed consent was obtained from all participants before the protocol began. 
After collecting demographic and deployment information, participants were assessed by a trained research assistant for the presence of psychiatric disorders with the Mini-International Neuropsychiatric Interview (MINI) (Lecrubier, 1997). PTSD symptoms were assessed with the Clinician Administered PTSD Scale (CAPS) (Blake, 1995), and ADHD symptoms were assessed with the Conners Adult ADHD Diagnostic Interview for DSM-IV (CAADID) (Conners, 1999). A board-certified psychiatrist (author initials) interviewed participants for combat exposure history and to confirm PTSD status per DSM-IV criteria, as well as other major psychiatric illnesses that would be exclusionary. Participants who met eligibility criteria were allowed to proceed with further assessments, including the Conners' Adult ADHD Rating Scales (CAARS) and a psychiatric clinical interview to determine ADHD diagnostic status. All measures were completed during a single visit to the clinical laboratory.

\section{Materials}

ADHD Symptoms: Conners' Adult ADHD Rating Scales-Self-Report: Short Version (CAARS-S:S). The CAARS-S:S (Conners, 1999) contains 26 items measuring symptoms of ADHD. Raw scale scores in the subscales (Inattention/Memory Problems; Hyperactivity/Restlessness; Impulsivity/Emotional Lability; Problems with Self Concept) are transformed into a standard T-score with a mean of 50 and a standard deviation of 10, normed by gender and age. T-scores above 65 indicate clinically significant symptoms.

PTSD Symptoms: Clinician Administered PTSD Scale (CAPS). The CAPS (Blake, 1995) is a gold standard diagnostic interview for current and lifetime PTSD and has excellent psychometric properties. Across samples, the CAPS demonstrates high inter-rater reliability (i.e., above .86) and internal consistency on each of the three PTSD symptom clusters (range .63 to .89), and correlates strongly (i.e., above .61) with other measures of PTSD. When tested in 
conjunction with the SCID, the CAPS provided a PTSD diagnosis with specificity ranging from $94 \%$ to $95 \%$, and sensitivity ranging from $84 \%$ to $90 \%$ (Radnitz, 1998; Hyer, 1996). Developed by researchers at the National Center of PTSD, this structured interview assesses all 17 symptoms of PTSD for frequency (scored on a 0 [never] to 4 [daily or almost every day]) and intensity (0 [none] to 4 [extreme, incapacitating distress]). These 17 symptoms can be totaled for a dichotomous diagnostic index or a continuous measure of posttraumatic stress severity, as well as DSM-related symptom cluster subscales (Reexperiencing, CAPS-B; Avoidance, CAPS-C; Hyperarousal, CAPS-D).

\section{Psychiatric Diagnoses: The MINI International Neuropsychiatric Interview and}

Chart Review. The MINI (Lecrubier, 1997) is a brief, valid, and reliable structured diagnostic interview for DSM-IV and ICD-10 psychiatric disorders. Participants were administered the MINI to screen for other Axis I disorders. When participants screened positive for a given diagnosis, study clinicians used follow up questions and record review to validate diagnoses and ensure eligibility criteria were met. Many participants met criteria for at least one psychiatric condition besides ADHD or PTSD. Co-occurring psychiatric conditions were most common in the ADHD+PTSD (73.1\%) and PTSD only (60.0\%) groups, followed by the ADHD only (29.4\%) group, and least common in controls (6.5\%). As shown in Table 1, depressive disorders were most common across groups; there was a significant difference in the proportion of participants with depression in each group $\left(X^{2}(3)=48.7, p<.001\right)$, with depression being more common in the PTSD only and ADHD+PTSD groups than the ADHD only and control group, and more common in the ADHD only group than in the control group.

Combat Exposure: Combat Exposure Scale (CES) (Lund, 1984) is a 7-item self-report measure, used to obtain information regarding exposure to wartime stressor events. The measure 
yields total scores ranging from 1 to 41 , where higher scores indicate greater severity of combat exposure.

Inhibitory Functioning. Inhibitory control (response inhibition and execution) was measured using a cued go/no-go $(\mathrm{GNG})$ reaction time task used in other research to measure inhibitory control in response to alcohol administration (Marczinski, 2005; Fillmore, 2009) and in adults and youth with ADHD (Weafer, 2009; Derefinko, 2008).

The task was operated using Inquisit software using a pre-programmed script for the Cued GNG task (Millisecond Software, Seattle, WA) and was performed on a PC. As described in the task user manual, each trial involved the following sequence. First, a fixation point $(+)$ was presented for $800 \mathrm{~ms}$ followed by a blank white screen for $500 \mathrm{~ms}$. Next, a visual cue was displayed for one of five stimulus onset asynchronies (SOAs $=100,200,300,400$, or $500 \mathrm{~ms}$ ). SOAs were distributed evenly across trials and varied to minimize anticipation effects. Following the cue, a go or no-go target stimulus was displayed until either a correct response occurred or $1000 \mathrm{~ms}$ had elapsed. Trials were separated by a $700 \mathrm{~ms}$ inter-trial interval.

Cues were rectangles framed in black and filled with white on a white background (appeared "empty"). Rectangles were either horizontal or vertical in orientation. Targets were presented as a solid fill to the interior of the rectangular cues. Go targets were green, and no-go targets were blue. Participants were instructed to respond on the keyboard spacebar with their right index finger as soon as a green (go) target appeared and to suppress a response when a blue (no-go) target was presented. The orientation of the cues signaled the probability of a subsequent go or no-go target. The orientation of the empty rectangles was a visual cue regarding the probability of a go vs. no-go response requirement. The vertical rectangle had an $80 \%$ probability of being a green go trial and the horizontal rectangle had an $80 \%$ probability of being 
a blue no-go trial. Cues with a high probability of correctly signaling a target were considered "valid cues" (vertical-green/go and horizontal-blue/no-go) and cues with a low probability of correctly signaling a target were considered "invalid cues" (vertical-blue/no-go and horizontalgreen/go).

Participants completed a total of 250 trials with equal distribution of vertical $(125 ; 100$ green/go, 25 blue/no-go) and horizontal cues (125; 100 blue/no-go, 25 green/go). For each trial, the computer recorded whether a response occurred, and if so, the reaction time (RT) in milliseconds from the onset of target to key press. Feedback (the words "correct" or "incorrect" along with RT) was presented during the inter-trial interval to promote quick and accurate responding.

Three primary measures were derived from the cued go/no-go task for this study. Inhibitory failures were measured as the proportion of no-go targets for which a participant failed to inhibit a response (i.e., responded to a no-go target). Scores were calculated for each cue condition (go and no-go). Group effects on proportion of inhibitory failures in the go and nogo cue conditions were analyzed by separate ANOVAs. Response execution was measured by RT to go targets. Shorter RTs indicated greater facilitation of response execution (Marczinski, 2005; Fillmore, 2009; Weafer, 2009; Derefinko, 2008). Mean RT scores were calculated for each participant for each cue type. Group effects on RT scores in the go and no-go cue conditions were analyzed by two ANOVAs. Omission errors were measured as non-responses to go targets. Omission errors were generally infrequent (typically $<2$ per session), which is consistent with previously reported findings in adults with ADHD (Weafer, 2009) and children with ADHD (Derefinko, 2008). Because no significant differences were observed between veteran and civilian participants on task performance variables, data were pooled for veterans and civilians 
within diagnostic group. Post-hoc group contrasts were conducted with Bonferroni or Tamhane's correction to account for multiple comparisons depending on whether there was homogeneity of variance across dependent variables by group.

\section{Results}

Failure of response inhibition following invalid (go) cues. Inhibitory failures were generally uncommon across all diagnostic groups (Table 2). Participants with co-occurring PTSD and ADHD demonstrated approximately twice as many inhibitory failures following invalid (go) cues as participants in the control group (2.3\% vs. 1.2\%), though the group effect was not significant, $F(3,156)=.74, p=.52, \eta_{\mathrm{p}}{ }^{2}=.014$

Failure of response inhibition following valid (no-go) cues. Participants in the control group demonstrated significantly fewer inhibitory failures following valid (no-go) cues relative to all three clinical groups, $F(3,156)=3.02, p=.03, \eta_{\mathrm{p}}{ }^{2}=.055$. There were no significant differences among the three clinical groups ( $p$-values all > .05).

Cue dependency. Cue dependency effects were most prominent in the control and PTSD+ADHD groups. Participants in the ADHD only and PTSD only groups demonstrated similar proportions of inhibitory failures to no-go trials regardless of cue condition.

Response execution and RT variability following valid (go) cues. There was a significant group effect on mean reaction time $\left(F(3,156)=8.62, p<.001, \eta_{\mathrm{p}}{ }^{2}=.142\right)$ and mean reaction time $\operatorname{SD}\left(F(3,156)=12.52, p<.001, \eta_{\mathrm{p}}^{2}=.194\right)$ when go cues were presented prior to the go target. Post hoc contrasts indicated that the ADHD+PTSD group responded significantly more slowly on those trials than the control group, $p=.03$ (Table 2). Additionally, the PTSD only $(p=$ $.01)$ and ADHD+PTSD groups $(p=.001)$ had more variable response times than the control group. 
Response execution and RT variability following invalid (no-go) cues. There was a significant group effect on mean reaction time $\left(F(3,156)=7.78, p<.001, \eta_{\mathrm{p}}{ }^{2}=.130\right)$ and mean reaction time $\operatorname{SD}\left(F(3,156)=7.52, p<.001, \eta_{\mathrm{p}}{ }^{2}=.126\right)$ when no-go cues were presented prior to the go target. Post hoc contrasts indicated that the PTSD only group $(p=.06)$ and the ADHD+PTSD group $(p=.06)$ responded marginally more slowly on those trials than the control group. Additionally, the PTSD only $(p=.04)$ and ADHD+PTSD groups $(p=.009)$ had significantly more variable response times than the control group.

Omission errors. Although overall rates of omission errors were very low, significant group effects were observed for both go cue $\left(F(3,156)=5.19, p=.002, \eta_{\mathrm{p}}{ }^{2}=.091\right)$ and no-go cue $\left(F(3,156)=5.58, p=.001, \eta_{\mathrm{p}}^{2}=.097\right)$ trials. Specifically, although no pairwise comparisons revealed significant differences, post-hoc contrasts indicated that the ADHD and ADHD+PTSD groups demonstrated significantly more omission errors than the control group following both go cues $(p=.010)$ and no-go cues $(p=.012)$.

\section{Discussion}

The purpose of this study was to evaluate behavioral indices of neurocognitive functioning - specifically response execution and inhibition - among veterans with and without ADHD and PTSD. Results indicated that participants with either PTSD or co-occurring ADHD+PTSD demonstrated significantly longer and more variable reaction times than controls. These effects generally held regardless of whether cues preceding the target stimuli were valid or invalid. Considered together, findings suggest that veterans with co-occurring ADHD+PTSD demonstrate slower response execution and impaired response inhibition similar to patterns reported in other samples of veterans and other groups with ADHD. Notably, co-occurring ADHD+PTSD was not associated with amplified deficits in these domains. There is some 
evidence that anxiety may attenuate or buffer impulsive responding in commonly observed in ADHD (Schatz, 2006). It is possible that anxiety or hypervigilance symptoms of PTSD - or comorbid depression, which was most common among participants with PTSD or ADHD+PTSD in this study - may have played a similar role, lengthening response time and lowering the risk of inhibitory failures among participants with ADHD+PTSD.

Participants with only ADHD did not differ significantly from participants with only PTSD or PTSD+ADHD on any outcome variable. However, they also did not differ from comparison participants upon post hoc analysis. This trend runs counter to prior findings in the literature where patients with ADHD performed worse (i.e., more inhibitory failures, more variable responding, etc.) than healthy controls. Even though a significant group difference was not observed between participants with ADHD and controls, the mean scores of the ADHD group were comparable to the ADHD+PTSD and PTSD groups. One reason for the lack of significant group effects between participants with ADHD only and controls was the overall smaller sample size and greater variability in the ADHD group. Some trend-level differences between the ADHD and control group were observed, but after correcting for multiple comparisons and non-homogeneity of variance between groups, these effects were not significant. It is possible this absence of group differences is the result of limited power, and significant differences might emerge in a study that enrolled more participants with ADHD only.

We did not observe significant differences in cue dependency across groups despite our prediction that participants with PTSD may be more sensitive to cues than participants without the disorder. Although some research has pointed the importance of cue-dependent learning in co-occurring PTSD and substance use disorders (Coffey, 2010; Saladin, 2003), it may be that effects are specific to particular types of cues or triggers, such as threat cues in the case of PTSD 
or cues that trigger craving in substance use. In the current study, the cues presented in the task were not linked to a particular rewarding or aversive outcome but rather to the likelihood of a particular type of target stimulus being presented in a given trial. It is possible that a cue dependency effect may be observed among participants with PTSD or ADHD+PTSD relative to participants without ADHD when cues not only predict likelihood of response expectation (press vs. not press) but if successful execution of those behaviors were linked to more salient consequences, such as aversive or rewarding stimuli contingent on performance.

The current study was the first to compare response execution and inhibition, as well cue dependency, in a sample of veterans and community adults with and without both ADHD and PTSD. Whereas other patterns of clinical comorbidity - such as PTSD and mood disorders, PTSD and substance use disorders, and ADHD and substance use disorders - have been studied more extensively, there is relatively little published research on ADHD+PTSD. Given that ADHD and PTSD are both common among military personnel and veterans, as well as in the general population though less frequently, additional research is needed to better understand the mechanisms underlying their comorbidity as well as the effectiveness of existing interventions designed for each disorder among people with both disorders.

Despite the contributions of the current study, a number of limitations should be noted. First, the study enrolled only males, due to relatively high rates of ADHD in males relative to females, higher rates of males served by the VA medical center, and the eligibility criteria of the parent study which involved genetic analyses. Future work should enroll female participants to evaluate for possible sex and gender effects. Second, rates of inhibitory failures and omission errors were low in this study. Low rates of errors have been reported on go/no-go tasks previously, especially among healthy adult control participants, but error rates in this study were 
lower than anticipated overall - particularly among the clinical groups. Although the cued go/no-go reaction time task we used here has been used in other studies (Weafer, 2009;

Derefinko, 2008), it is possible that the task was not sensitive to differences in inhibitory functioning in the current sample, particularly outside the context of alcohol challenge as has been investigated previously. Additional studies in other groups, including larger samples with co-occurring ADHD, mood, and anxiety disorders - as well as in younger samples of children and adolescents - may help clarify the impact of comorbidity on impulsive responding in ADHD. Future studies should include multiple indices of inhibitory functioning, including versions of go/no-go or stop tasks with more stringent response requirements or that include more salient consequences to motivate faster responding across trials. The current study was also limited in its capacity to account for other factors that may have influenced task performance, such as personality, history of head injury, or substance use disorder. 


\section{Acknowledgements}

This work was supported by the [masked] VA Medical Center, and the Clinical Sciences Program of the Department of Veterans Affairs (Merit Review grants [masked] and [masked] awarded to Dr. [masked]), and federal grants [masked] (to Dr. [masked]) and [masked] (supporting Dr. [masked]). The authors also wish to thank [masked] for his assistance in preparing data sets for analysis. The views expressed in this article are those of the author(s) and do not necessarily represent the views of the Department of Veterans Affairs. All research performed was in compliance with the code of ethics of the World Medical Association (Declaration of Helsinki) and the standards established by the [masked] VA Medical Center Institutional Review Board. 
Running head: RESPONSE INHIBITION IN MEN WITH PTSD/ADHD

\section{Financial Disclosures}

The authors reported no biomedical financial interests or potential conflicts of interest. 


\section{References}

Adler, L. A., Kunz, M., Chua, H. C., Rotrosen, J., \& Resnik, S. G. (2004). Attentiondeficit/hyperactivity disorder in adult patients with posttraumatic stress disorder (PTSD): Is ADHD a vulnerability factor. Journal of Attention Disorders, 8(1), 11-16.

American Psychiatric Association. (2013). Diagnostic and statistical manual of mental disorders: DSM-5. Washington, D.C: American Psychiatric Association.

Antshel, K. M., Kaul, P., Biederman, J., Spencer, T. J., Hier, B. O., Hendricks, K., \& Faraone, S. V. (2013). Posttraumatic stress disorder in adult attention-deficit/hyperactivity disorder. Journal of Clinical Psychiatry, 74, E197-E204.

Barkley, R. A. (1997). Behavioral inhibition, sustained attention, and executive functions: Constructing a unifying theory of ADHD. Psychological Bulletin, 121(1), 65-94.

Blake, D. D., Weathers, F. W., Nagy, L. M., Kaloupek, D. G., Gusman, F. D., Charney, D. S., \& Keane, T. M. (1995). The development of a clinician-administered PTSD scale. Journal of Traumatic Stress, 8(1), 75-90.

Bremner, J. D. (1999). Does stress damage the brain? Biological Psychiatry, 45, 797-805.

Breslau, N. (2009). The epidemiology of trauma, PTSD, and other posttrauma disorder. Trauma, Violence, \& Abuse, 10(3), 198-210.

Buckley, T. C., Blanchard, E. B., \& Neill, W. T. (2000). Information processing and PTSD: A review of the empirical literature. Clinical Psychology Review, 20, 1041-1065.

Coffey, S. F., Schumacher, J. A., Stasiewicz, P. R., Henslee, A. M., Baillie, L. E., \& Nandy, N. (2010). Craving and physiological reactivity to trauma and alcohol cues in posttraumatic stress disorder and alcohol dependence. Experimental \& Clinical Psychopharmacology, $18,340-349$. 
Conners, C. K., Erhardt, D., Epstein, J. N., Parker, J. D. A., Sitarenios, G., \& Sparrow, E. (1999). Self-ratings of ADHD symptoms in adults I: Factor structure and normative data. Journal of Attention Disorders, 3(3), 141-151.

Derefinko, K. J., Adams, Z. W., Milich, R., Fillmore, M. T., Lorch, E. P., \& Lynam, D. R. (2008). Response style differences in the inattentive and combined subtypes of attentiondeficit/hyperactivity disorder. Journal of Abnormal Child Psychology, 36(5), 745-758.

Falconer, E., Bryant, R., Felmingham, K. L., Kemp, A. H., Gordon, E., Peduto, A., ... Williams, L. M. (2012). The neural networks of inhibitory control in posttraumatic stress disorder. Journal of Psychiatry \& Neuroscience, 33, 413-422.

Fillmore, M. T., Ostling, E. W., Martin, C. A., \& Kelly, T. H. (2009). Acute effects of alcohol on inhibitory control and information processing in high and low sensation-seekers. Drug \& Alcohol Dependence, 100(1), 91-99.

Hanson, J. A., Haub, M. D., Walker, J. J., Johnston, D. T., Nelson Goff, B. S., \& Dretsch, M. N. (2012). Attention deficit hyperactivity disorder subtypes and their relation cognition functioning, mood states, and combat stress symptomatology in deploying U.S. soldiers. Military Medicine, 177, 655-662.

Harrington, K. M., Miller, M. W., Wolf, E. J., Reardon, A. F., Ryabchenko, K. A., \& Ofrat, S. (2012). Attention-deficit/hyperactivity disorder comorbidity in a sample of veterans with posttraumatic stress disorder. Comprehensive Psychiatry, 53, 679-690.

Hyer, L., Summers, M. N., Boyd, S., Litaker, M., \& Boudewyns, P. (1996). Assessment of older combat veterans with the Clinician-Administered PTSD Scale. Journal of Traumatic Stress, 9(3), 587-593. 
Jovanovic, T., Ely, T., Fani, N., Glover, E. M., Gutman, D., Tone, E. B., ... Ressler, K. J. (2013). Reduced neural activation during an inhibition task is associated with impaired fear inhibition in a traumatized civilian sample. Cortex, 49, 1884-1891.

Jovanovic, T., Kazama, A., Bachevalier, J., \& Davis, M. (2012). Impaired safety signal learning may be a biomarker of PTSD. Neuropharmacology, 62, 695-704.

Kertzman, S., Avital, A., Weizman, A., \& Segal, M. (2014). Intrusive trauma recollections is associated with impairment of interference inhibition and psychomotor speed in PTSD. Comprehensive Psychiatry, 55, 1587-1594.

Kessler, R. C., Adler, L., Barkley, R., Biederman, J., Conners, C. K., Demler, O., . . Zaslavsky, A. M. (2006). The prevalence and correlates of adult ADHD in the United States: Results from the National Comorbidity Survey Replication. American Journal of Psychiatry, 163, 716-723.

Khaylis, A., Polusny, M. A., Erbes, C. R., Gewitz, A., \& Rath, M. (2011). Posttraumatic stress, family adjustment, and treatment preferences among national guard soldiers deployed to OEF/OIF. Military Medicine, 176, 126-131.

Lecrubier, Y., Sheehan, D. V., Weiller, E., Amorim, P., Bonora, I., Sheehan, K. H., ... \& Dunbar, G. C. (1997). The Mini International Neuropsychiatric Interview (MINI). A short diagnostic structured interview: reliability and validity according to the CIDI. European Psychiatry, 12(5), 224-231.

Lund, M., Foy, D., Sipprelle, C., \& Strachan, A. (1984). The Combat Exposure Scale: a systematic assessment of trauma in the Vietnam War. Journal of Clinical Psychology, 40(6), 1323-1328. 
Marczinski, C. A. \& Fillore, M. T. (2005). Alcohol increases reliance on cues that signal acts of control. Experimental and Clinical Psychopharmacology, 13(1), 15-24.

Morein-Zamir, S., Dodds, C., van Hartevelt, T. J.,Schwarzkopf, W., Sahakian, B., Muller, U., \& Robbins, T. (2014). Hypoactivation in right inferior frontal cortex is specifically associated with motor response inhibition in adult ADHD. Human Brain Mapping, 35, $5141-5152$.

Myers, C. E., VanMeenen, K. M., \& Servatius, R. J. (2012). Behavioral inhibition and PTSD symptoms in veterans. Psychiatry Research, 196, 271-276.

O’Connell, R. G., Bellgrove, M. A., Dockree, P. M., Lau, A., Hester, R., Garavan, H., Fitzgerald, M., Foxe, J. J., \& Robertson, I. H. (2009). The neural correlates of deficient error awareness in attention-deficit hyperactivity disorder (ADHD). Neuropsychologia, 47, 1149-1159.

Oosterlan, J., Logan, G. D., Sergeant, J. A. (1998). Response inhibition in AD/HD, CD, comorbid $\mathrm{AD} / \mathrm{HD}+\mathrm{CD}$, anxious, and control children: A meta-analysis of studies with the stop task. Journal of Child Psychology \& Psychiatry, 39(3), 411-425.

Pittman, J. O. E., Goldsmith, A. A., Lemmer, J. A., Kilmer, M. T., \& Baker, D. G. (2012). Posttraumatic stress disorder, depression, and health-related quality of life in OEF/OIF veterans. Quality of Life Research, 21, 99-103.

Radnitz, C. L., Hsu, L., Tirch, D. D., Willard, J., Lillian, L. B., Walczak, S., ... \& Schlein, I. (1998). A comparison of posttraumatic stress disorder in veterans with and without spinal cord injury. Journal of Abnormal Psychology, 107(4), 676. 
Roberts, W., Milich, R., \& Fillmore, M. T. (2016). The effects of preresponse cues on inhibitory control and response time in adults with ADHD. Journal of Attention Disorders, 20(4), $317-324$.

Saladin, M. E., Drobes, D. J., Coffey, S. F., Dansky, B. S., Brady, K. T., and Kilpatrick, D. G. (2003). PTSD symptom severity as a predictor of cue-elicited drug craving in victims of violent crime. Addictive Behaviors, 28(9), 6-29.

Schatz, D. B., \& Rostain, A. L. (2006). ADHD with comorbid anxiety a review of the current literature. Journal of Attention Disorders, 10(2), 141-149.

Sebastian, A., Gerdes, B., Feige, B., Kloppel, S., Lange, T., Philipsen, A., van Elst, L. T. Lieb, K., \& Tuscher, O. (2012). Neural correlates of interference inhibition, action withholding and action cancelation in adult ADHD. Psychiatry Resesarch, 202, 132-141.

Simmonds D, Pekar J, Mostofsky S (2008) Meta-analysis of Go/No-go tasks demonstrating that fMRI activation associated with response inhibition is task-dependent. Neuropsychologia, 46, 224-232.

Simon, V., Czobar, P., Bálint, S., Mésáros, Bitter, I. (2009). Prevalence and correlates of adult attention-deficit hyperactivity disorder: Meta-analysis. British Journal of Psychiatry, 194, 204-211.

Swick, D., Honzel, N., Larsen, J., \& Ashley, V. (2013). Increased response variability as a marker of executive dysfunction in veterans with post-traumatic stress disorder. Neuropsychologia, 51, (14), 3033-3040.

Tillman, G. D., Green, T. A., Ferree, T. C., Calley C. S., Maguire, M. J., Briggs, R., Hart, J., Haley, R. W., \& Kraut, M. A. (2010). Impaired response inhibition in ill Gulf War veterans. Neurological Science, 297, 1-5. 
Van Rooij, S. J. H., Rademaker, A. R., Kennis, M., Vink, M., Kahn, R. S., \& Geuze, E. (2014). Impaired right inferior frontal gyrus response to contextual cues in male veterans with PTSD during response inhibition. Journal of Psychiatry \& Neuroscience, 39, 330-338.

Van Rooij, S. J. H., Geuze, E., Kennis, M., Rademaker, \& A. R., Vink. (2015). Neural correlates of inhibition and contextual cue processing related to treatment response in PTSD. Neuropsychopharmacology, 40, 667-675.

Weafer, J., Fillmore, M. T., \& Milich, R. (2009). Increased sensitivity to the disinhibiting effects of alcohol in adults with ADHD. Experimental \& Clinical Psychopharmacology, 17(2), 113.

Woltering, S., Liu, Z., Rokeach, A., \& Tannock, R. (2013). Neurophysiological differences in inhibitory control between adults with ADHD and their peers. Neuropsycholgia, 51, 1888-1895.

Table 1. Demographic and clinical information by group

\begin{tabular}{|c|c|c|c|c|c|c|}
\hline Variable & $\begin{array}{c}\text { ADHD only } \\
(n=17)\end{array}$ & $\begin{array}{l}\text { PTSD only } \\
\quad(n=25)\end{array}$ & $\begin{array}{l}\text { ADHD + } \\
\text { PTSD } \\
(n=26)\end{array}$ & $\begin{array}{l}\text { Control } \\
(n=92)\end{array}$ & $F$ & $\begin{array}{l}\text { Chi- } \\
\text { square }\end{array}$ \\
\hline $\begin{array}{l}\text { Age, years } \\
M(S D)\end{array}$ & $32.4(7.5)$ & $32.3(7.0)$ & $33.8(8.0)$ & $29.3(.76)$ & $3.21^{*}$ & -- \\
\hline Race (\% white) & 79.6 & 66.7 & 82.3 & 65.4 & -- & 29.1 \\
\hline $\begin{array}{l}\text { Combat } \\
\text { Exposure Scale }\end{array}$ & $20.3(13.5)$ & $18.5(7.6)$ & $23.6(8.3)$ & $17.0(8.9)$ & 2.43 & -- \\
\hline CAPS-Total & $\begin{array}{c}35.8(12.2)^{\mathrm{a}} \\
(\mathrm{n}=8)\end{array}$ & $65.1(9.2)^{b}$ & $68.7(10.9)^{b}$ & $\begin{array}{c}30.2(15.1) \\
\mathrm{a}\end{array}$ & $\begin{array}{c}54.9 * * \\
*\end{array}$ & \\
\hline
\end{tabular}




$$
(n=21)
$$

CAPS-B

$$
\begin{aligned}
& 6.1(5.1)^{\mathrm{a}} \quad 15.6(6.1)^{\mathrm{b}} \quad 15.8(5.6)^{\mathrm{b}} \quad 5.4(4.2)^{\mathrm{a}} \quad 21.3^{* *} \\
& (\mathrm{n}=8) \quad(\mathrm{n}=21) \quad *
\end{aligned}
$$

CAPS-C

$$
\begin{aligned}
& 12.4(8.7)^{\mathrm{a}} \quad 26.3(7.6)^{\mathrm{b}} \quad 28.2(6.0)^{\mathrm{b}} \quad 11.7(8.2)^{\mathrm{a}} \quad 26.6^{* *} \\
& (n=8) \\
& (\mathrm{n}=21) \quad *
\end{aligned}
$$

CAPS-D

$$
\begin{aligned}
& 17.3(4.6)^{\mathrm{a}} \quad 23.2(4.3)^{\mathrm{b}} \quad 24.8(4.1)^{\mathrm{b}} \quad 13.1(7.0)^{\mathrm{a}} \quad 24.4^{* *} \\
& (n=8) \\
& (n=21)
\end{aligned}
$$

CAARS-A

$61.2(12.7)^{\mathrm{a}} \quad 48.6(8.5)^{\mathrm{b}} \quad 66.8(8.9)^{\mathrm{a}} \quad 44.7(7.4)^{\mathrm{b}} \quad 55.9^{* *}$

CAARS-B

$62.2(8.3)^{\mathrm{a}} \quad 52.9(6.7)^{\mathrm{a}}$

$60.7(6.9)^{\mathrm{a}}$

$46.2(9.0)^{\mathrm{b}} \quad 32.5^{* *}$

CAARS-C

$55.8(11.5)$

$51.5(9.7)^{\mathrm{b}} \quad 60.7(9.1)^{\mathrm{a}}$

$42.9(5.8)^{\mathrm{c}} \quad 43.0^{* *}$

$\mathrm{ab}$

CAARS-D

$52.5(12.6)^{\mathrm{a}} \quad 53.4(11.0)$

$64.7(9.3)^{b}$

$42.9(7.3)^{\mathrm{c}} \quad 43.5^{* *}$

a

$*$

CAARS-E

$60.6(10.1)^{\mathrm{a}} \quad 51.5(7.6)^{\mathrm{b}}$

$67.5(5.9)^{\mathrm{c}}$

$42.8(7.1)^{\mathrm{d}} \quad 90.3 * *$

Disorder (\%)

Adjustment

0.0

20.0

7.7

1.1

$16.1 * *$

Antisocial

$8.5 *$

Personality

5.9

0.0

0.0

0.0

Anxiety

5.9

24.0

11.5

3.3

$11.9 * *$

Bipolar

0.0

4.0

7.7

0.0 
Borderline

Personality

0.0

0.0

3.8

1.1

$48.7 * *$

Depression

29.4

48.0

57.7

4.3

Insomnia

0.0

4.0

0.0

0.0

Intermittent

Explosive

0.0

0.0

3.8

0.0

Panic

0.0

8.0

7.7

0.0

$8.7 *$

Social anxiety

0.0

0.0

7.7

1.1

5.9

Note: ADHD: Attention-deficit/hyperactivity disorder. PTSD: Posttraumatic stress disorder. CAPS: Clinician Administered PTSD Scale. CAPS-B: Reexperiencing scale. CAPS-C: Avoidance scale. CAPS-D: Hyperarousal scale. CAARS: Connors Adults ADHD Rating Scale. CAARS-A: Inattention/Memory Problems. CAARS-B: Hyperactivity/Restlessness. CAARS-C: Impulsivity/Emotional Lability. CAARS-D: Problems with Self-Concept. CAARS-E: ADHD Index. $* p<.05 . * * p<.01 . * * * p<.001$. Co-occurring psychiatric diagnoses were determined by a combination of scores on the MINI screening interview and follow-up question and record review by study clinicians. Values with different superscript letters were significantly different per post hoc contrast analysis, $p<.05$, following correction for multiple comparisons (Bonferroni or Tamhane, depending on whether equal variances observed). Values that share a superscript did not differ significantly from each other. Values without superscripts were not significantly different from any other values. 
Table 2. Cued Go/No-Go Task performance and reaction time data across groups.

\begin{tabular}{lccccc}
\hline Variable & $\begin{array}{c}\text { ADHD only } \\
(n=17)\end{array}$ & $\begin{array}{c}\text { PTSD only } \\
(n=25)\end{array}$ & $\begin{array}{c}\text { ADHD+PTSD } \\
(n=26)\end{array}$ & $\begin{array}{c}\text { Control } \\
(n=92)\end{array}$ & F \\
\hline Proportion IF & $1.4 \%$ & $1.4 \%$ & $2.3 \%$ & $1.2 \%$ & .74 \\
Go Cue & & & & & $3.02 *$ \\
Proportion IF & $1.5 \%$ & $1.2 \%$ & $1.2 \%$ & $0.5 \%$ & \\
No-Go Cue & & & & & \\
Cue & -0.1 & 0.2 & 1.1 & 0.7 &
\end{tabular}

dependency

$\begin{array}{lllll}\text { Reaction } \quad 353.4(102.9) & 343.1(63.7) & 382.4(122.7)^{\mathrm{a}} & 307.6(38.2)^{\mathrm{b}} & 8.62^{* * * *}\end{array}$

time Go Cue

Reaction $\quad 358.2(103.6) \quad 354.1(67.0)^{\mathrm{a}} \quad 385.7(125.7)^{\mathrm{a}} \quad 315.3(34.9)^{\mathrm{b}} \quad 7.78^{* * *}$

time No-Go

Cue

Reaction $\quad 80.8(45.6) \quad 78.6(35.3)^{\mathrm{a}} \quad 90.0(42.2)^{\mathrm{a}} \quad 52.9(23.3)^{\mathrm{b}} \quad 12.52^{* * * *}$

time SD Go-

Cue

$\begin{array}{lllll}\text { Reaction } & 75.0(53.3) & 74.1(40.7)^{\mathrm{a}} \quad 75.7(36.3)^{\mathrm{a}} & 49.4(24.9)^{\mathrm{b}} & 7.52^{* * *}\end{array}$

time SD No-

Go Cue

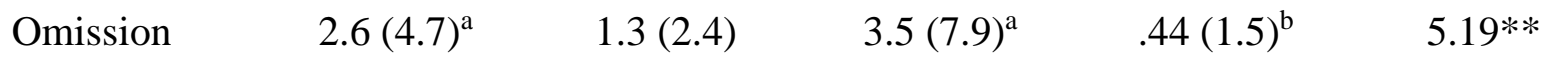

errors Go

Cue 
Running head: RESPONSE INHIBITION IN MEN WITH PTSD/ADHD

$\begin{array}{lllll}\text { Omission } & .65(1.2)^{\mathrm{a}} \quad .20(.41) & 1.1(2.6)^{\mathrm{a}} & .07(0.3)^{\mathrm{b}} & 5.58 * * \\ \text { errors No-Go } & \end{array}$

\section{Cue}

Note. IF = inhibitory failures. $\mathrm{SD}=$ standard deviation. Values with different superscript letters were significantly different per post hoc contrast analysis with Tamhane's correction applied, $p<$ $.05 . * p<.05 . * * p<.01 . * * * p<.001$. Values that share a superscript did not differ significantly from each other. Values without superscripts were not significantly different from any other values. 
